\title{
EFICIENCIA DE UN TRATAMIENTO SUPERFICIAL CON NANOPARTÍCULAS COMO OPCIÓN DE MANTENIMIENTO INTEGRAL DE ESTRUCTURAS DE CONCRETO
}

\author{
F. TIENDA, J. CANINO, D. CRUZ-MORENO, L. VACA-ARCIGA, G. FAJARDO, B. LIMÓN \\ Universidad Autónoma de Nuevo León, Facultad de Ingeniería Civil, San Nicolás de los Garza, Nuevo León, México
}

\begin{abstract}
RESUMO
El objetivo de este trabajo fue evaluar la eficiencia de un tratamiento superficial con nanopartículas base silicio funcionalizadas (NF) como opción de mantenimiento e incremento en la durabilidad del concreto. Para esto se fabricaron especímenes de concreto que se expusieron a carbonatación y al crecimiento de Clorella sp. Una vez alcanzado el grado de exposición definido, se aplicó una suspensión con $0.1 \%$ de NF. Posteriormente, los especímenes se expusieron nuevamente para evaluar el desempeño del tratamiento mediante seguimiento de la profundidad de carbonatación, ángulo de contacto, crecimiento de biomasa por peso seco y microscopía electrónica de barrido. Los resultados mostraron que las condiciones de exposición en laboratorio sobrepasaron la capacidad de protección del tratamiento. No obstante, bajo condiciones reales, se logró inhibir el crecimiento de microorganismos y reducir el avance de la carbonatación.
\end{abstract}

Palabras clave: concreto; durabilidad; nanopartículas; tratamiento superficial.

\begin{abstract}
The objective of this project was to evaluate the efficiency of a functionalized silica-based nanoparticles (NF) surface treatment as a maintenance option and the increase in concrete durability. For this reason, concrete samples were made to be exposed under carbonation and Chlorella sp. growth. Once reached the defined level of exposure, a suspensión with $0.1 \%$ de NF was applied. Later, the samples were exposed again to evaluate the treatment's performance through the continuous measurement of the carbonation advance, wáter contact angle, biomass growth and scanning electron microscopy. The results indicate that laboratory exposure conditions exceed the treatment protection capacity. Nevertheless, under real condition, it achieved the micrsorganisms growth inhibition and reduce the carbonatation advance.
\end{abstract}

Keywords: concrete; durability; nanoparticles; surface treatment.

\section{RESUMO}

O objetivo deste trabalho foi avaliar a eficiência de um tratamento superficial com nanopartículas funcionalizadas à base de silício (NF) como opção de manutenção e incremento da durabilidade do concreto. Para isso, foram fabricados corpos de prova de concreto que foram expostos à carbonatação e ao crescimento de Clorella sp., respectivamente. Uma vez alcançado o grau de exposição definido, foi aplicada uma suspensão com $0,1 \%$ de NF. Em seguida, os corpos de prova foram novamente expostos para avaliar o desempenho do tratamento com testes de profundidade de carbonatação, ângulo de contato e microscopia eletrônica de varredura. Os resultados mostraram que as condições de exposição no laboratório excederam a capacidade de proteção do tratamento. No entanto, sob condições reais, foi possível inibir o crescimento de microorganismos e reduzir o avanço da carbonatação.

Palavras-chave: concreto; durabilidade; nanopartículas; tratamento superficial. 


\section{INTRODUCCIÓN}

Existe un problema en la industria de la construcción, específicamente en las estructuras de concreto reforzado. Esto se debe al elevado porcentaje de estructuras que, por falta de mantenimiento, deficiente durabilidad en los materiales, un erróneo proceso constructivo, una exposición ante agentes agresivos, etc., se encuentran en una situación donde es necesario realizar reparaciones. O bien, han llegado a condiciones deplorables y no pueden continuar en servicio. Todo esto, genera un gran impacto económico que lo vuelve un área de interés primordial.

Es importante desarrollar e implementar nuevos procedimientos y/o técnicas que incorporen nuevos materiales que puedan brindar una solución a tales problemas. Para ello, es necesario asegurar que dichas alternativas sean factibles y eficientes. Actualmente, el uso de nanopartículas se ha introducido a la industria de la construcción, como una opción para mejorar las propiedades de los materiales. El uso y efecto de las nanopartículas base silicio ha propiciado que se exploren diversas líneas de investigación donde se apliquen y puedan resolver carencias que presentan los materiales de construcción en su desempeño. Se ha demostrado que las nanopartículas base silicio a través de una funcionalización pueden generar un efecto hidrofóbico en la superficie de los materiales base cemento portland (Cruz-Moreno et. al, 2016; DeFerri et. al, 2011).

El ingreso de humedad es un factor que influye en la durabilidad de los materiales, ya que permite que tomen lugar fenómenos de envejecimiento de las estructuras construidas con concreto reforzado u otro material base cemento. Por ello, las nanopartículas base silicio funcionalizadas (NF) podrían usarse como un tratamiento en estructuras que se encuentren en servicio y necesiten de algún mantenimiento que pueda incrementar su durabilidad o garantizar el tiempo de vida para el cual fue proyectada o diseñada.

Existen dos condiciones de envejecimiento que aquejan a las estructuras de concreto reforzado: la carbonatación y el crecimiento de microorganismos. La carbonatación es un fenómeno generado a causa del ingreso de dióxido de carbono en la matriz cementante del concreto, el cual reacciona con la humedad interna y transforma el hidróxido de calcio en carbonato de calcio. En estructuras de concreto sin acero de refuerzo, este fenómeno tiene pocas consecuencias, pero en el concreto armado promueve la corrosión del acero, que genera pérdida de resistencia mecánica en el acero y puede producir fracturas en el concreto (Possan, 2010).

Por otro lado, el crecimiento de microorganismos es un fenómeno que puede generar daños estéticos y el posterior deterioro del material, al metabolizar o descomponer el área afectada por la biomasa (Márquez, 2015). Dependiendo del caso y del tipo de microorganismo que se generen, pueden ser dañinos para salud de los usuarios. El crecimiento de microorganismos es un problema común en las estructuras de concreto gracias a la porosidad. Debido a que sólo necesita de humedad y nutrientes como nitrógeno y fósforo, por lo que es muy fácil que se presente en cualquier superficie de material base cemento expuesta a la intemperie. En este trabajo se determinó evaluar el crecimiento de Chlorella $s p$., una microalga con amplia presencia en ambientes húmedos y que además es común encontrarla en consorcios de microorganismos como el moho y los líquenes (Márquez, 2015). Se optó por el uso de esta microalga por la facilidad de manipulación y las condiciones de crecimiento y nutrientes fáciles de brindarse.

Dichos agentes de deterioro o envejecimiento se consideraron por ser muy comunes en la región, debido a las condiciones de exposición en las que se encuentran la mayoría de las obras. Usualmente, el moho y los líquenes promueven un mayor biodeterioro en los materiales, debido a las distintas actividades metabólicas que se llevan a cabo por parte de los hongos, las microalgas y/o las bacterias que se encuentren en dicho consorcio (Márquez, 2015). Por esto es de interés evaluar de manera aislada uno de estos componentes, como la Chlorella sp. para analizar su crecimiento ante el uso de nanopartículas base silicio funcionalizadas (NF). 


\section{DESARROLLO EXPERIMENTAL}

La experimentación se sintetiza en la obtención de especímenes con cierto grado de envejecimiento, posterior a ello la aplicación de los tratamientos evaluados y, finalmente, una evaluación de éstos ante una reexposición a los medios de envejecimiento. En cada una de las etapas se realizó una caracterización física y/o química de los materiales utilizados, para mostrar las características bajo las cuales se realizaron las distintas actividades y así pudieran ser aplicadas en investigaciones futuras.

\subsection{Caracterización y obtención de especímenes}

Se diseñó el concreto con una relación agua cemento de 0.65 y un f'c=300 kg/ $\mathrm{cm}^{2}$ a 28 días, utilizando el método del ACI318-2014, obteniendo el proporcionamiento presentado en la Tabla 1. Se realizaron mezclas de prueba para controlar los procesos de mezclado y lograr estandarizarlo y disminuir los errores al momento de elaborar las mezclas para las distintas series de especímenes.

Tabla 1. Proporcionamiento del concreto utilizado para la fabricación de especímenes.

\begin{tabular}{|c|c|}
\hline Material & Cantidad $(\mathbf{k g} / \mathbf{1 0 0 0 L})$ \\
\hline Cemento (CPO40) & 330.0 \\
\hline Agua & 239.9 \\
\hline A. grueso (TMA $=9.5 \mathrm{~mm})$ & 756.1 \\
\hline A. fino & 918.6 \\
\hline Suma & $2,244.6$ \\
\hline
\end{tabular}

Para caracterizar el concreto se realizaron pruebas de revenimiento (17.5 cm, ASTM C143), masa unitaria $\left(2.1 \mathrm{~kg} / \mathrm{m}^{3}\right.$, ASTM C138), contenido de aire (1.5\%, ASTM C173), temperatura $\left(22^{\circ} \mathrm{C}\right.$, ASTM 1064) y una vez que fraguaron los especímenes, se realizaron pruebas para determinar la resistencia mecánica (Figura 1.) (ASTM C39).

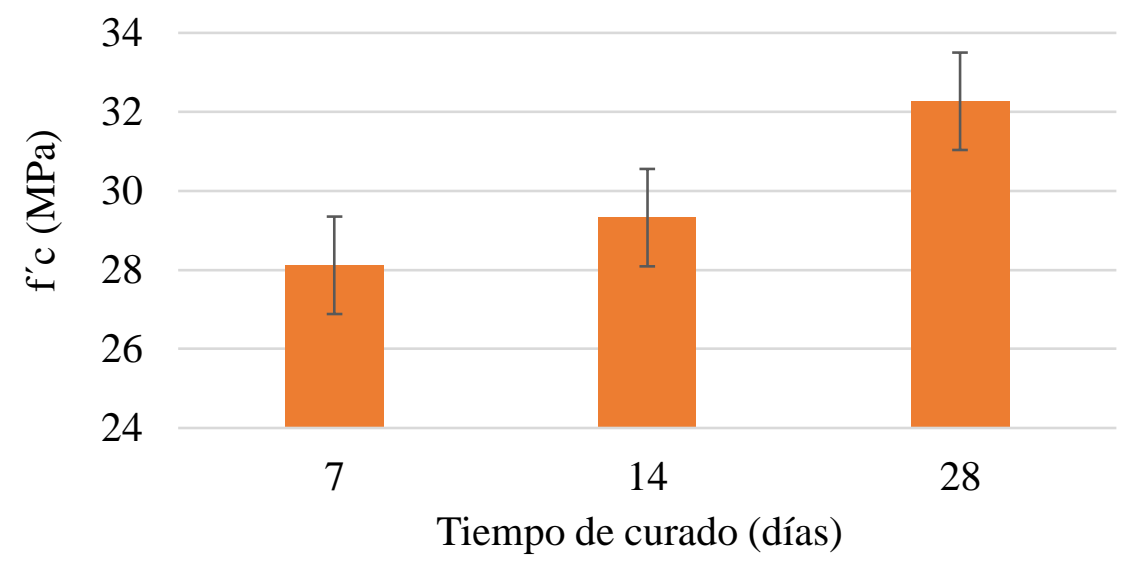

Figura 1. Desarrollo de la resistencia a compresión del concreto fabricado (ASTM C39).

\subsection{Síntesis de las nanopartículas}

Las NF se obtuvieron mediante una síntesis sol-gel, a $70^{\circ} \mathrm{C}$. Se usó tetraetilortosilicato (TEOS: (Si(OC2H5)4) como precursor de silicio, alcohol etílico (C2H6O al 95\%), e hidróxido de amonio $(\mathrm{NH} 4 \mathrm{OH})$, para su funcionalización se adicionaron 1,1,3,3- tetrametildisiloxano y ácido nítrico para su modificación superficial. Este procedimiento puede ser consultado con mayor detalle en trabajos previos (Cruz-Moreno, 2014). 


\subsection{Obtención de Chlorella sp. y fabricación del biorreactor}

Para preparar la suspensión a utilizar en la experimentación se tomó un cultivo de Chlorella $s p$. obtenida y caracterizada por el laboratorio de Ingeniería Ambiental, IIC, UANL. La muestra de Chlorella sp. (conservada en un estado de hibernación a $4^{\circ} \mathrm{C}$ ) fue activada una vez que se incrementó la temperatura, se le brindó luz y un medio nutritivo.

Se inició con $200 \mathrm{ml}$ de biomasa (suspensión concentrada con el cultivo aislado), luego se adicionaron $200 \mathrm{ml}$ de medio nutritivo BG11 que se mantuvo con un periodo de luz de 12 horas al día y 12 horas de oscuridad, a $22 \pm 2{ }^{\circ} \mathrm{C}$ y un suministro de aire. Para esto, se utilizó una bomba de aire para forzar la entrada de aire al fondo del recipiente y evitar la sedimentación de las microalgas. Durante este último proceso, las microalgas obtenían el carbono necesario del $\mathrm{CO}_{2}$ contenido en el aire introducido.

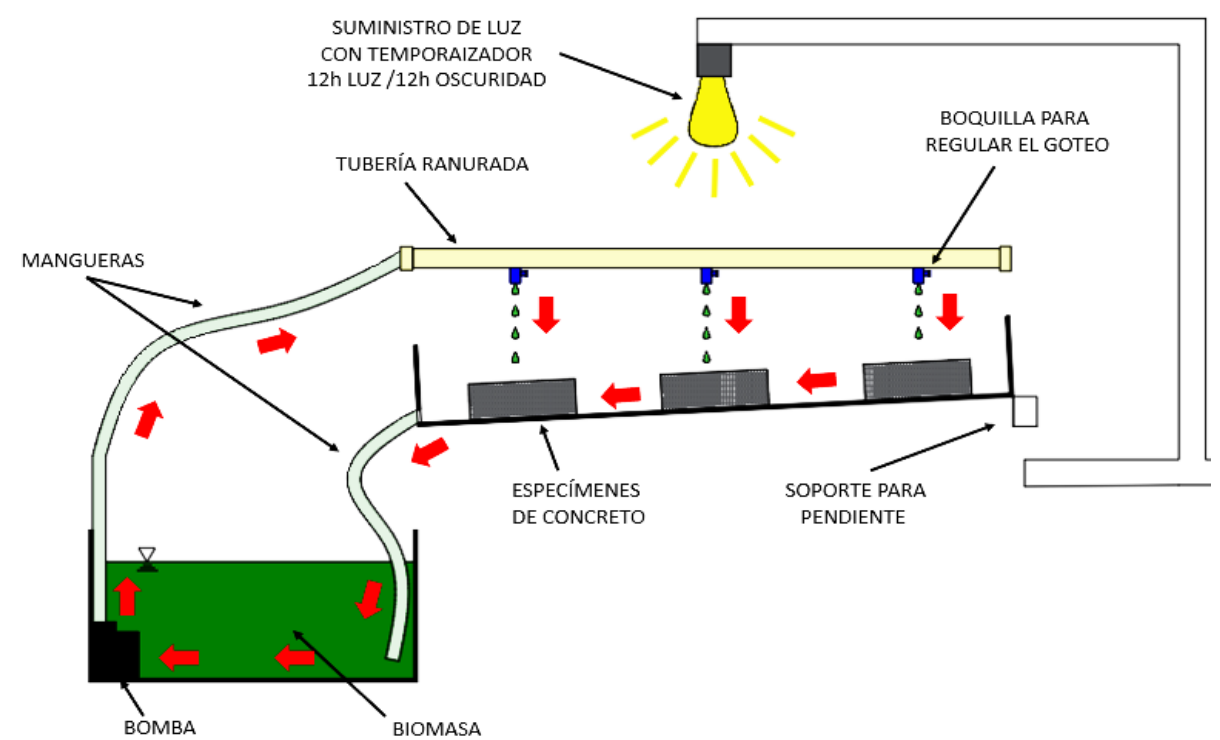

Figura 2. Esquema del biorreactor de flujo continuo fabricado para la experimentación del proyecto de tesis de licenciatura (Tienda, 2019).

Así se continuó el proceso adicionando agua y BG11 hasta obtener el volumen necesario para el biorreactor. Una vez que se tuvo el volumen adecuado de la suspensión se colocó en un biorreactor de flujo continuo que se fabricó y que se esquematiza en la Figura 2. En este caso, no fue necesaria la bomba de aire, ya que en las áreas de goteo el sistema estuvo expuesto al aire por lo que la interacción con el $\mathrm{CO}_{2}$ se dio libremente.

\subsection{Fabricación de especímenes y medios de exposición 2.4.1Carbonatación}

Para los especímenes sometidos a envejecimiento por carbonatación se fabricaron prismas de concreto de 10x10x30 cm. Éstos, luego de desmoldarse, se pusieron en curado a una H.R. $=100 \%$ durante 28 días. Posterior a ello, se sacaron del curado se dejaron secar al medio ambiente. Una vez secos, se recubrieron sus bases con un sello e impermeabilizante para delimitar la penetración de carbonatación sólo a las demás paredes. Una vez preparados los especímenes se introdujeron a una cámara de carbonatación con una concentración de $15 \%$ de $\mathrm{CO}_{2}$, una H.R. $=70 \%$ y a $22 \pm 2^{\circ} \mathrm{C}$. Ahí permanecieron hasta alcanzar la profundidad de carbonatación de 0 o $5 \mathrm{~mm}$ respectivamente.

Para la determinación de la profundidad de la carbonatación en los elementos de concreto se realizaron cortes de manera transversal, para exponer el interior del material. Posteriormente, se aplicó una solución con fenolftaleína para evidenciar la reducción del $\mathrm{pH}$ debido al efecto de carbonatación (Figura 3) y así determinar cuándo iniciar con la aplicación del tratamiento según lo planeado. 


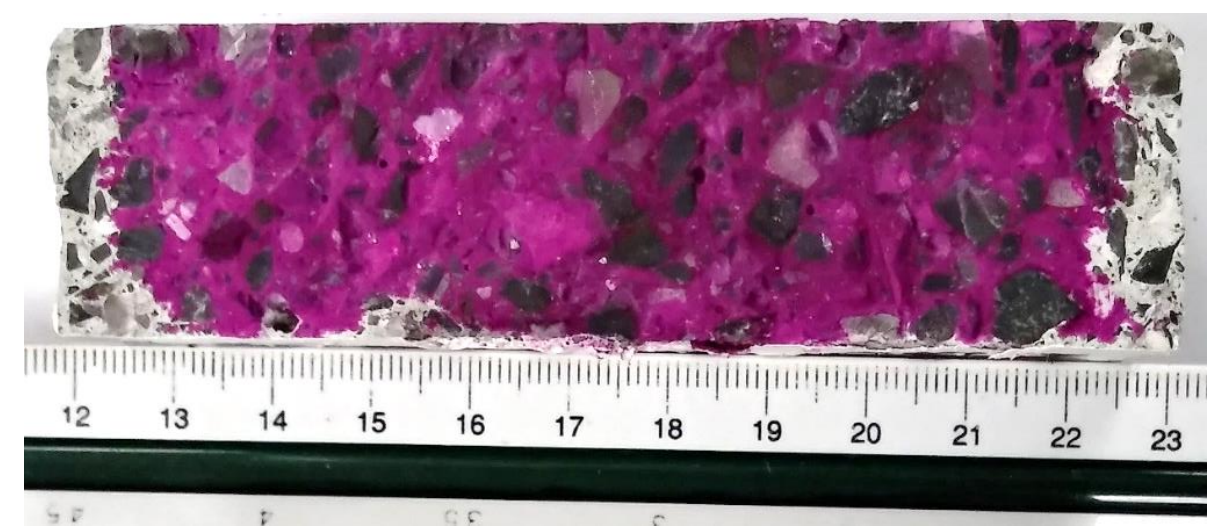

Figura 3. Imagen de una muestra de concreto posterior al rocío de la fenolftaleína.

\subsubsection{Microalgas (Chlorella sp.)}

Se fabricaron especímenes cilíndricos de concreto de $5 \mathrm{~cm}$ de diámetro y $13 \mathrm{~cm}$ de altura, los cuales fueron desmoldaron al día siguiente y posteriormente puestos en curado sumergidos en agua durante 14 días. Posterior al curado, cada cilindro fue seccionado de manera transversal en 4 partes, resultando muestras de una altura de $3 \mathrm{~cm}$.

Las muestras obtenidas de los especímenes se introdujeron a una cámara de carbonatación con una concentración de $\mathrm{CO}_{2}$ del $15 \%$, una $\mathrm{HR}$ de $70 \%$ y a $20 \pm 2^{\circ} \mathrm{C}$, con la finalidad de reducir la alcalinidad en los especímenes y así acelerar el crecimiento de las microalgas en su superficie. Cuando los especímenes alcanzaron una penetración por carbonatación de $2 \mathrm{~mm}$ fueron trasladados al biorreactor de flujo continuo para ser expuestos al cultivo de Chlorella sp. durante 0 y 14 días.

\subsubsection{Experimento in situ}

Con la finalidad de obtener una evaluación bajo condiciones reales se aplicó el tratamiento en un elemento expuesto a la intemperie. Para ello, el tratamiento con NF fue aplicado en la superficie del concreto de una rampa para discapacitados cubriendo una zona de $1 \times 1.2 \mathrm{~m}$. Después de 11 meses se extrajeron 2 núcleos de la rampa, uno de la zona tratada y otro de la zona a la cual no se le aplicó el tratamiento. Es necesario mencionar que, al momento de aplicar el tratamiento, la rampa ya contaba con 9 años de edad, aproximadamente.

Para evaluar el efecto que tuvo el tratamiento se hicieron distintas pruebas. Se aplicó fenolftaleína para medir la profundidad por carbonatación y se midió el ángulo de contacto para evaluar la permanencia del efecto hidrofóbico. También, se realizaron observaciones mediante microscopía electrónica de barrido para obtener la morfología de las muestras, confirmar la presencia de microorganismos y el estado de la matriz cementante. Finalmente, se determinó la biomasa por peso seco para medir de manera cuantitativa la cantidad de materia orgánica existente en las superficies.

\subsection{Aplicación de los tratamientos}

Una vez que las condiciones de envejecimiento establecidas fueron obtenidas en los especímenes, se prosiguió a aplicar las NF.

Primeramente, se limpió la superficie de cada elemento para que estuviera libre de polvo y manchas, para esto se utilizó agua destilada y un cepillo. Luego, se preparó una suspensión con agua destilada y $\mathrm{NF}$ al $0.1 \% \mathrm{~m} / \mathrm{v}$. No fue necesario aumentar la temperatura para la preparación y la mezcla se realizó a temperatura ambiente. Ya lista la solución, se colocó en un recipiente con un atomizador y se roció homogéneamente sobre toda la superficie de estudio del espécimen, se dejó secar 30 min o hasta que se observó una tonalidad mate, momento en que se procedió a la aplicación de una segunda capa de la suspensión. Luego de aplicación del tratamiento se dejaron 14 días en condiciones de laboratorio para que las nanopartículas pudieran tener un eficaz efecto en la superficie de los especímenes. 


\section{MÉTODOS DE EVALUACIÓN}

\subsection{Profundidad de Carbonatación}

El seguimiento del nivel de carbonatación provee un valor directo de cuán resistente o vulnerable son los especímenes tratados, en comparación con los no tratados, ante el fenómeno de carbonatación.

Para realizar las mediciones con fenolftaleína, se hicieron cortes transversales de $3 \mathrm{~cm}$ de espesor. Esto se realizó para cada que el espécimen alcanzaba una edad de evaluación ante la reexposición, es decir, se extrajeron de la cámara de carbonatación y se cortó una muestra, de cada espécimen, a los 12, 19, 26 y 33 días. La muestra de 10x10x3 cm se partía por la mitad con un cincel y en la parte de la fractura se aplicaba la fenolftaleína. De cada uno de estos 4 puntos se tomaban 4 mediciones y finalmente se promediaban todos los datos obtenidos para así tener el nivel de carbonatación de los especímenes a cada cierto tiempo de reexposición.

\section{2 Ángulo de Contacto}

El ángulo de contacto es una propiedad superficial materiales sólidos, que evalúa la adherencia que tiene un líquido a él y de este modo su tendencia a la hidrofobicidad, como se muestra en la clasificación de la Tabla 2.

Tabla 2. Clasificación de hidrofobicidad según el ángulo de contacto del agua (Fernández-Cañete, 2013).

\begin{tabular}{|c|c|}
\hline Hidrófilo & $\theta<90^{\circ}$ \\
\hline Hidrófobo & $\theta>90^{\circ}$ \\
\hline Super hidrófobo & $\theta>150^{\circ}$ \\
\hline
\end{tabular}

Para realizar esta medición se utilizaron los mismos especímenes usados para la carbonatación luego de hacer las mediciones con fenolftaleína y los especímenes expuestos a Chlorella sp. La parte evaluada fue en la superficie donde había sido aplicado el tratamiento.

Para hacer las mediciones se utilizó un goniómetro de la marca KRÜSS Drop Shape Analyzer modelo D5A25E. Las mediciones realizadas se procesaron con el software ADVANCE for Drop Shape Analyzers versión 1.9-03 2014-2018.

\subsection{Biomasa determinada por peso seco}

El crecimiento de las microalgas se midió retirando la biopelícula y cuantificando su peso. Para conseguirlo, se sumergieron los especímenes en una solución con $\mathrm{NaOH}(0.2 \mathrm{~g} / \mathrm{L})$ durante 24 h, cada uno en un frasco por separado. En este lapso la biopelícula se retiraba y la suspensión con la biomasa se filtraba utilizando microfiltros, estos filtros eran pesados antes de usarse. Para el filtrado se utilizó una bomba de vacío que servía para succionar la suspensión y hacer filtrar todo el líquido. El tiempo de filtrado variaba dependiendo la concentración de la biomasa, desde $1 \mathrm{~min}$ hasta $15 \mathrm{~min}$. Luego del filtrado, los filtros se colocaban en una estufa de laboratorio y se secaban a $105^{\circ} \mathrm{C}$, durante $4-6$ horas, hasta alcanzar un peso constante. Una vez secos los filtros, se volvieron éstos a pesar, con una precisión de $0.0001 \mathrm{~g}$ utilizando una balanza analítica. El peso de la biomasa se determinó por diferencia de pesos entre el filtro sólo y el filtro seco con biomasa retenida.

\subsection{Microscopía electrónica de barrido}

Las muestras evaluadas por MEB se obtuvieron únicamente de los especímenes que se expusieron al cultivo de Chlorella sp. y el experimento in situ. Todas por igual recibieron la misma preparación. Se cortaron los especímenes a un tamaño de 5x5x5 mm aproximadamente. 
Luego, se sumergieron en etanol y se metieron al horno a $60^{\circ} \mathrm{C}$ durante 3 días. Una vez secas las muestras, se llevaron a la recubridora de oro-paladio modelo Denton Vacuum - Desk V, la cual consiste en una cámara de vacío; la cual, al llegar a cierta presión, detecta la condición óptima de secado para aplicar el recubrimiento. Posterior a esto se realizó la prueba de MEB, para lo cual se utilizó un microscopio electrónico de barrido JEOL - JSM-6510LV.

\section{RESULTADOS}

\subsection{Influencia de la condición inicial de la superficie} 4.1.1 Carbonatación
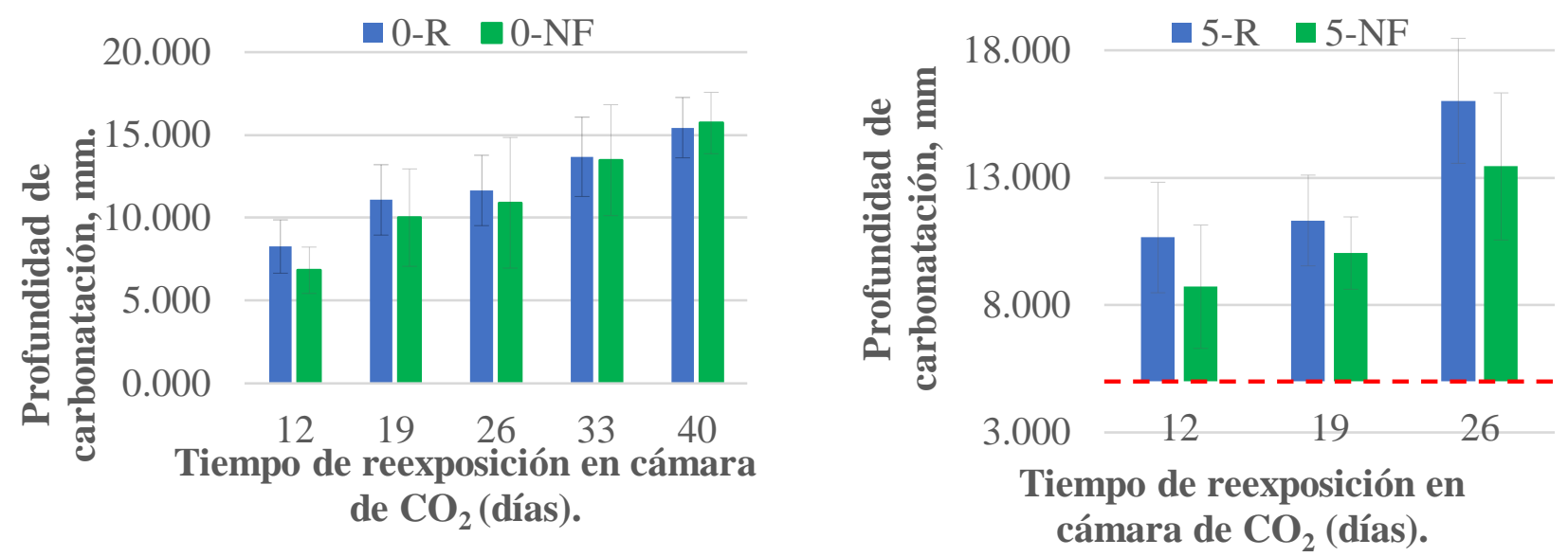

Figura 5. Nivel de carbonatación con respecto al tiempo de reexposición a $\mathrm{CO}_{2}$ (Especímenes envejecidos $0 \mathrm{~mm}$ izquierda y envejecidos $5 \mathrm{~mm}$ derecha)

En la Figura 5 se observa la profundidad de carbonatación con respecto al tiempo de exposición. Se puede ver que el avance de carbonatación es menor en los especímenes envejecidos a $5 \mathrm{~mm}$, que en los de $0 \mathrm{~mm}$. Esto se puedo apreciar desde los primeros 12 días, en los que el primero alcanza hasta $8 \mathrm{~mm}$ de profundidad, mientras que el otro llega hasta $6 \mathrm{~mm}$, si se comparan sus profundidades iniciales. Esta situación puede atribuirse a la densificación del concreto, propia de la carbonatación. Puede esperarse que, a mayor carbonatación sea más lento su avance, debido a que el ingreso de $\mathrm{CO}_{2}$ y humedad será más complicado. En la serie de $0 \mathrm{~mm}$ es posible apreciar que periódicamente el incremento de la carbonatación es menor, lo cual coincide con la idea anterior.

La previa carbonatación, aunado al uso del tratamiento que inhibe el ingreso de humedad dio lugar a un mejor desempeño en los especímenes envejecidos. Por otro lado, el inevitable avance de la carbonatación pudo suscitarse a causa de la humedad interna del material que no pudo ser expulsada, ante la inexistencia de un gradiente de temperatura y de humedad dentro de la cámara.

En la Figura 6 se observa el ángulo de contacto con respecto al tiempo en los especímenes expuestos a carbonatación. Se puede observar que la exposición acelerada provocó una reducción en el ángulo de contacto del agua a tal punto de estar en los límites para clasificarse como un sustrato hidrofóbico. En este sentido, la exposición a la carbonatación podría ser un factor que influya negativamente en el tratamiento con NF. 

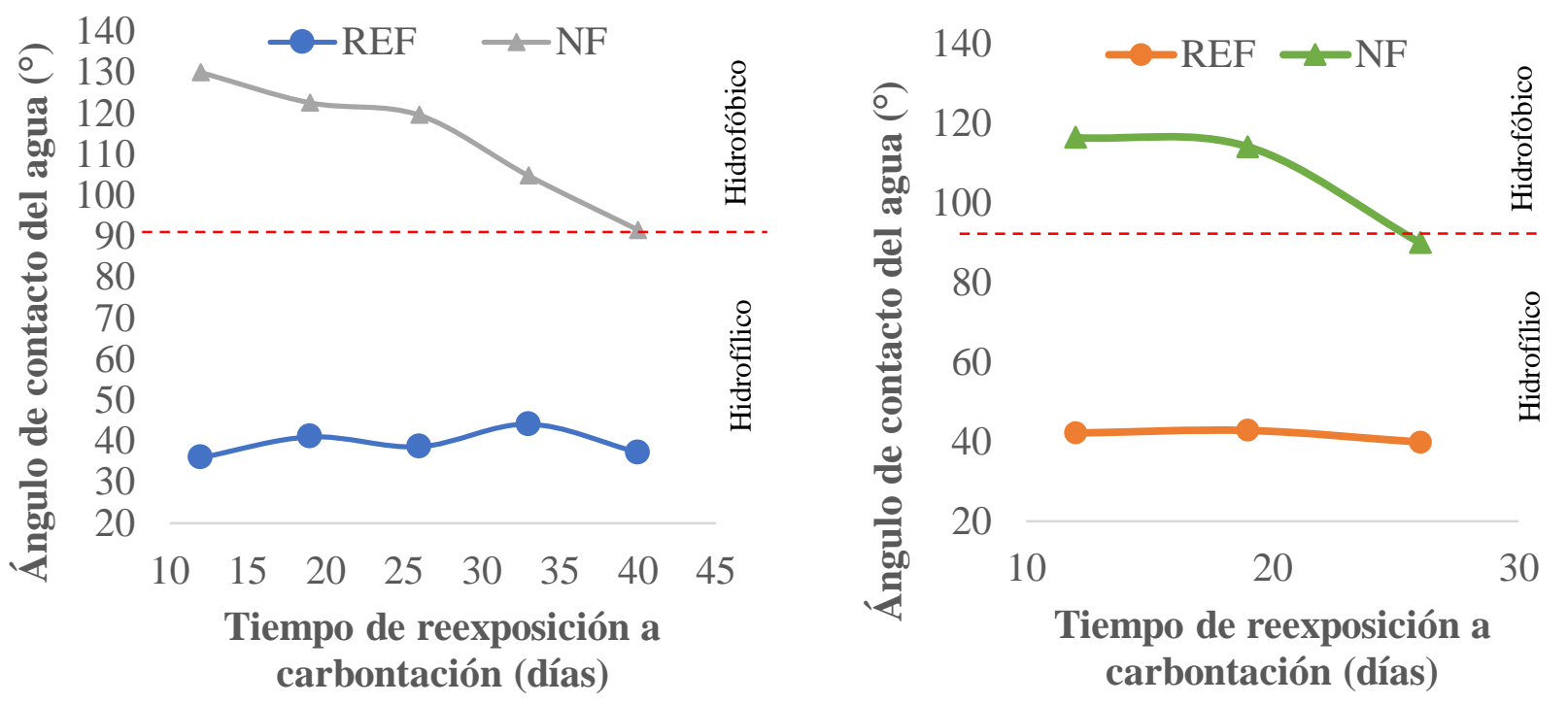

Figura 6. Ángulo de contacto en especímenes expuestos a carbonatación (Especímenes envejecidos $0 \mathrm{~mm}$ izquierda y envejecidos $5 \mathrm{~mm}$ derecha).

\subsubsection{Chlorella sp.}

En la Figura 7 se presenta la biomasa adherida en los especímenes expuestos a Chlorella sp., cantidad determinada por peso seco. Se percibe una menor adherencia de microalgas en los especímenes tratados, y esto se atribuye al efecto hidrofóbico que reduce la humedad contenida en la superficie de los especímenes.

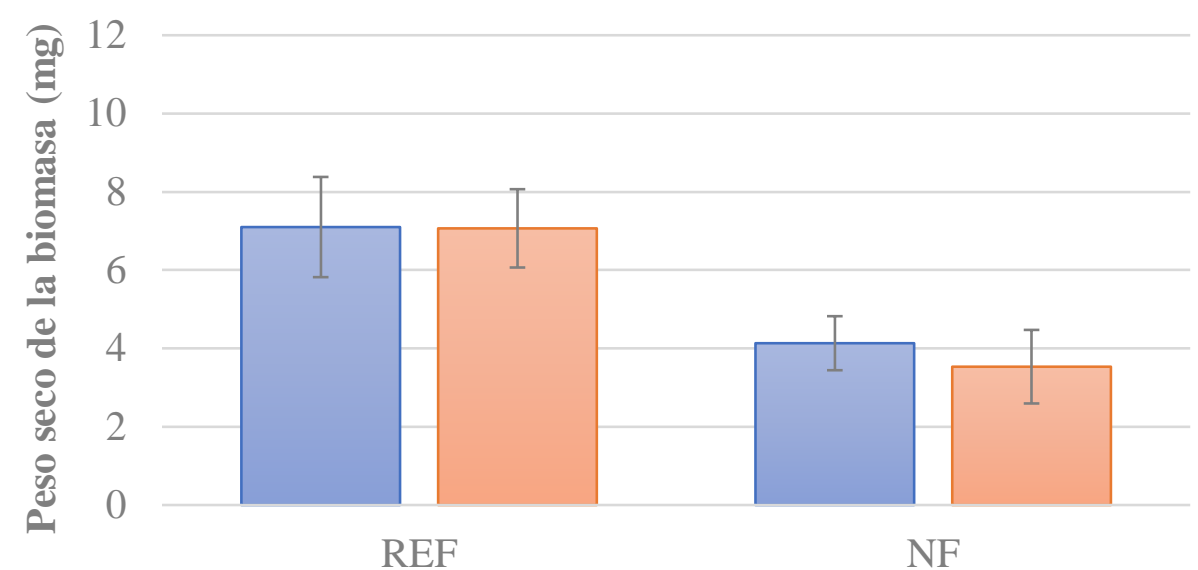

$\square 0$ días de envejecimiento $\square 14$ días de envejecimiento

Figura 7. Biomasa adherida en especímenes con 14 días de reexposición al cultivo de Chlorella sp.

Como se observa en la Figura 8, donde se muestra el ángulo de contacto del agua en los especímenes expuestos a Chlorella sp., se aprecia que existe una gran pérdida en el ángulo de contacto a tal punto que, luego de 14 días en el biorreactor, todos los tratamientos se encuentran por debajo o en los límites de $90^{\circ}$. Es decir que, el efecto hidrofóbico se ha perdido la mayoría de los casos. Motivo por el cual el crecimiento y adherencia de la Chlorella sp., a la superficie de las muestras, es posible. 
La reducción del efecto hidrofóbico sobre el crecimiento de los microorganismos puede deberse al gran porcentaje de humedad que se tenía. Debido al goteo, se generaban acumulaciones de agua sobre el sustrato que, aunque no estuvieran completamente adheridas, ahí se mantenían por largos periodos. Por lo tanto, se deduce que, el medio tan húmedo propició la disminución del efecto hidrofóbico de los tratamientos. Además, ninguno de los compuestos del tratamiento o de concreto (luego de la carbonatación) son biocidas, inclusive la sílice podría considerarse como un micronutriente de la Chlorella sp. Por lo cual, al estar bajo condiciones tan críticas, se logró vencer el efecto hidrofóbico y las microalgas se adaptaron al medio.
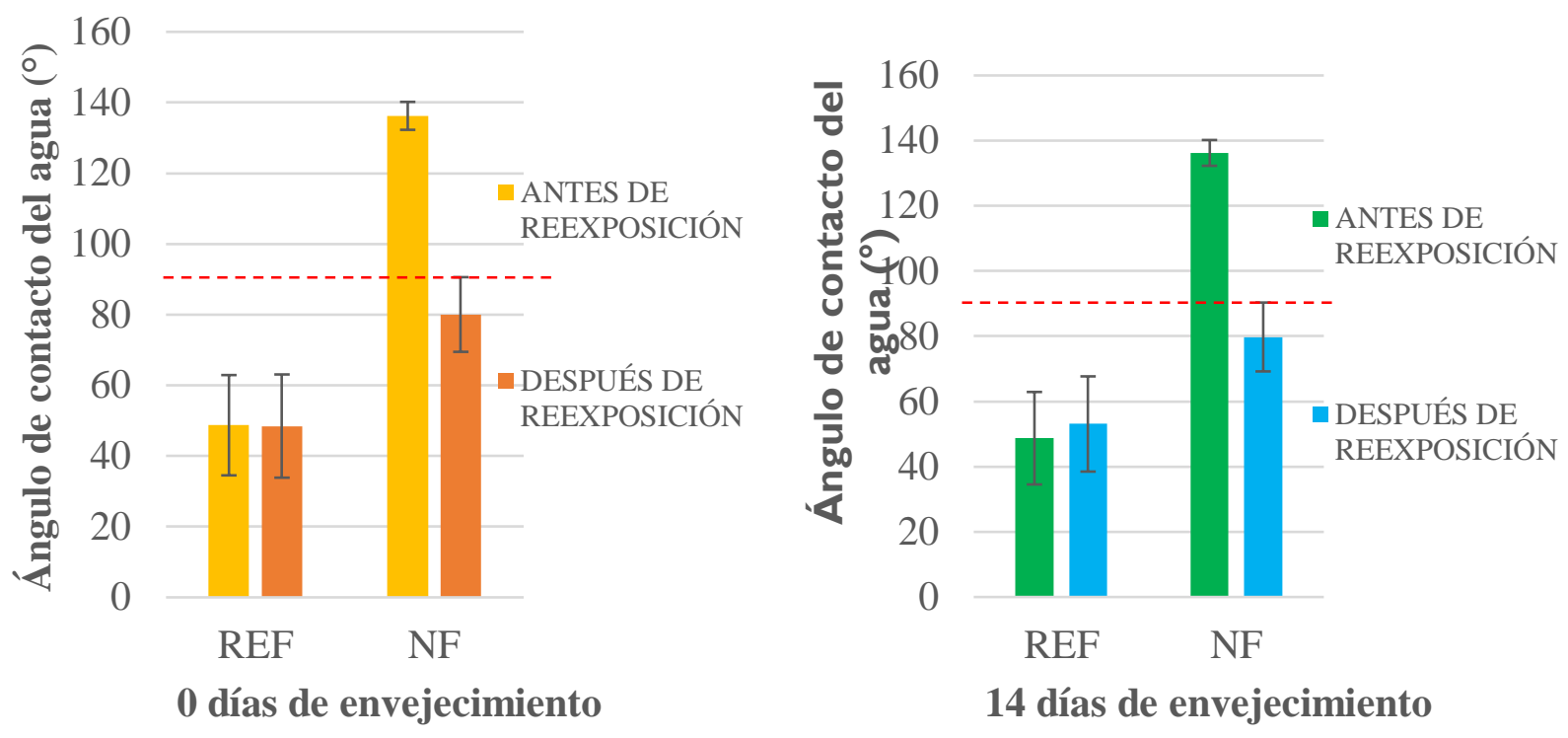

Figura 8. Ángulo de contacto del agua en los especímenes expuestos al crecimiento de Chlorella sp.

A pesar de que bajo las condiciones de exposición se propició un notable crecimiento independientemente del tratamiento aplicado, es posible identificar en las micrografías diferencias en cada uno de los tratamientos y que se relacionan con las demás pruebas realizadas.

En la Figura 9 se muestran las micrografías obtenidas de los especímenes expuestos a Chlorella $s p$. con 0 días de envejecimiento previo y se aprecia que en la muestra de referencia se observa la diferencia entre la biopelícula generada del lado izquierdo y la matriz cementante en el lado derecho, esto es posible notarlo por un desprendimiento de la biopelícula. Es preciso decir que le la biopelícula se nota más uniforme a diferencia de la muestra tratada donde es perceptible la morfología de las microalgas por una menor concentración y aglomeración de éstas.

En la Figura 10 se observa la diferencia de concentración de biomasa por la tonalidad que presenta la biopelícula, en este caso la muestra de referencia es más oscura que la tratada y ambas muestran una capa más notable que los especímenes sin envejecimiento previo.

La presencia de una concentración mayor en los especímenes con 14 días de envejecimiento, en comparación a los de 0 días, puede adjudicarse a dos factores:

1.- Los especímenes con un envejecimiento de 14 días fueron los últimos en ser expuestos en el biorreactor. Por lo tanto, el cultivo contenía células más maduras y, quizá en su momento, una mayor concentración de biomasa.

2.- La previa exposición (envejecimiento durante 14 días) pudo propiciar que las microalgas hayan generado un acondicionamiento de la superficie que facilitara el futuro crecimiento de Chlorella $s p$. 


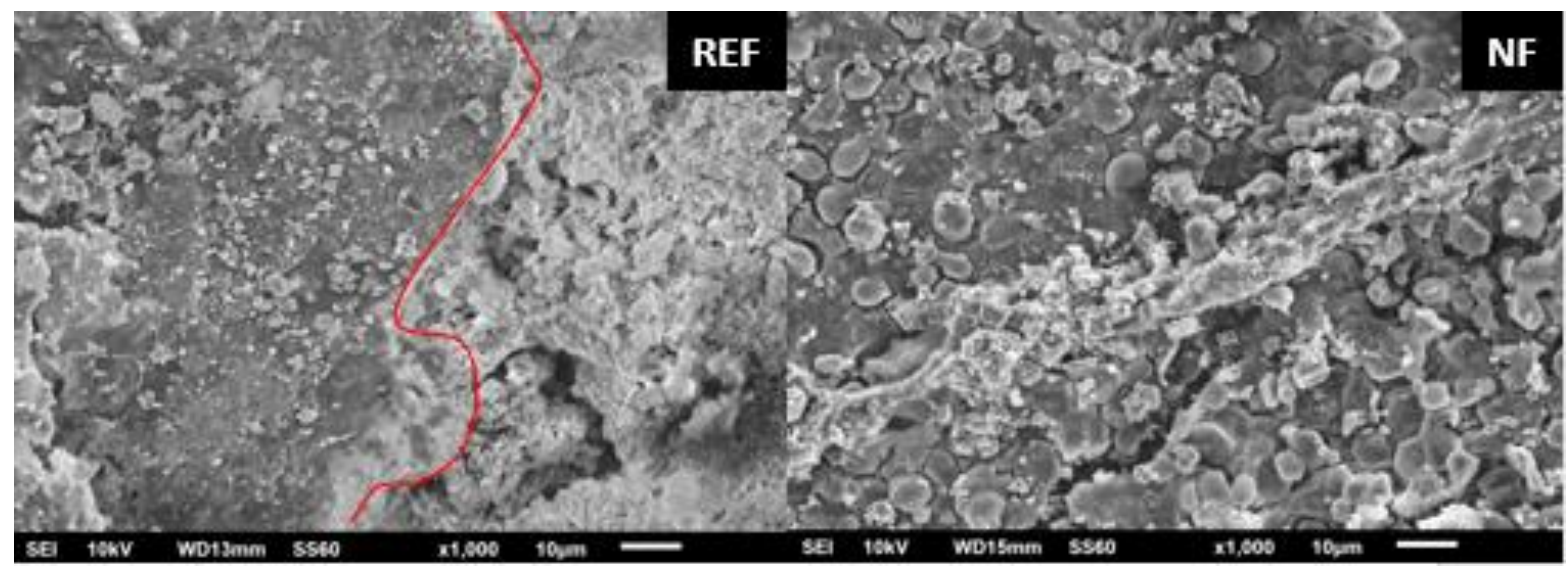

Figura 9. MEB de especímenes, con 0 días de envejecimiento, expuestos a Chlorella sp. por 14 días (1000x).

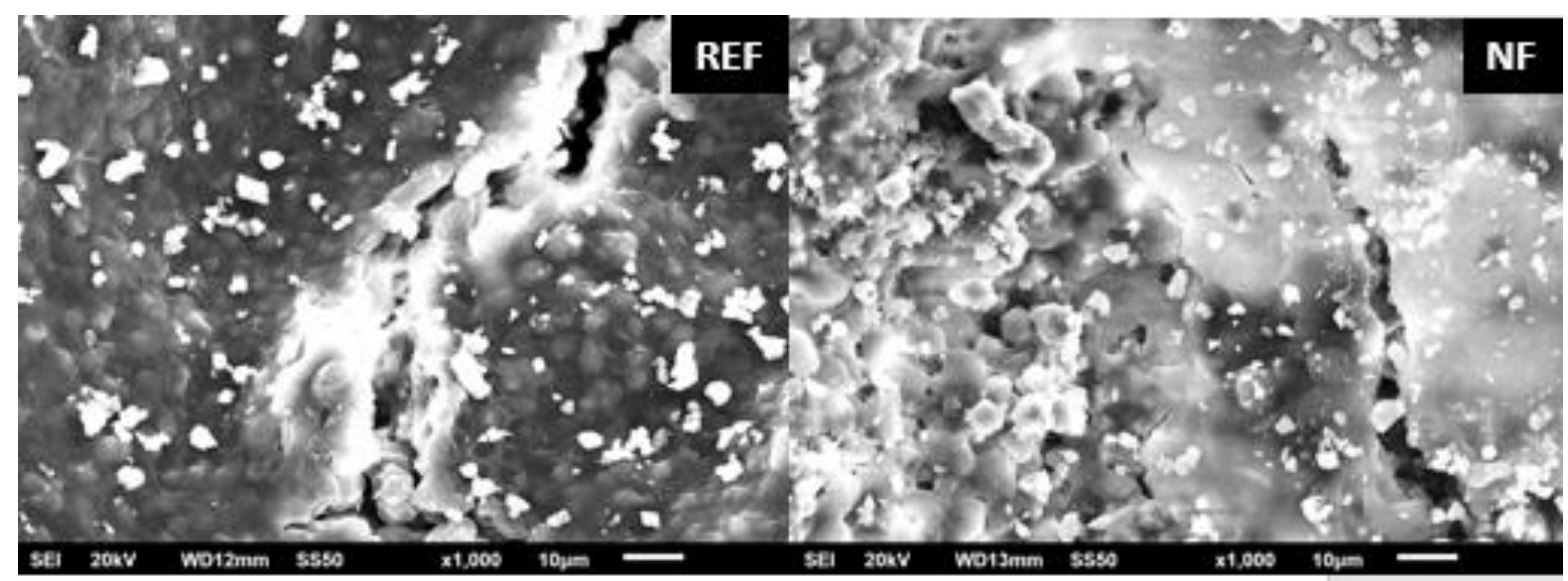

Figura 10. MEB de especímenes, con 14 días de envejecimiento, expuestos a Chlorella sp. por 14 días (1000x).

\subsection{Experimento in situ}

Luego de realizar un análisis en el Microscopio Electrónico de Barrido (Figura 11), de las muestras extraídas de la rampa, se pudo observar en la superficie del núcleo de referencia la presencia de lo que parece ser algún tipo de hongo, debido a sus estructuras alargadas, como ramificaciones, inclusive se aprecia la formación de una biopelícula en la superficie pues el nivel de colonización era muy notable.

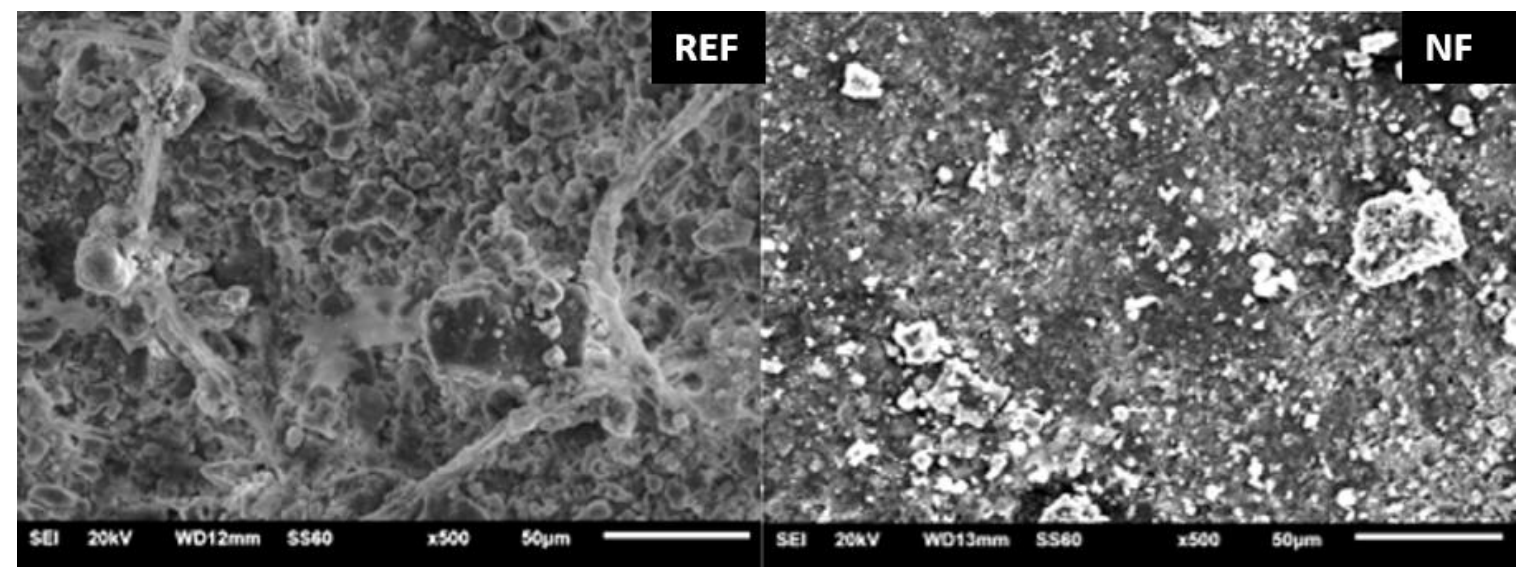

Figura 11. MEB de especímenes, con 14 días de envejecimiento, expuestos a Chlorella sp. por 14 días $(500 x)$. 


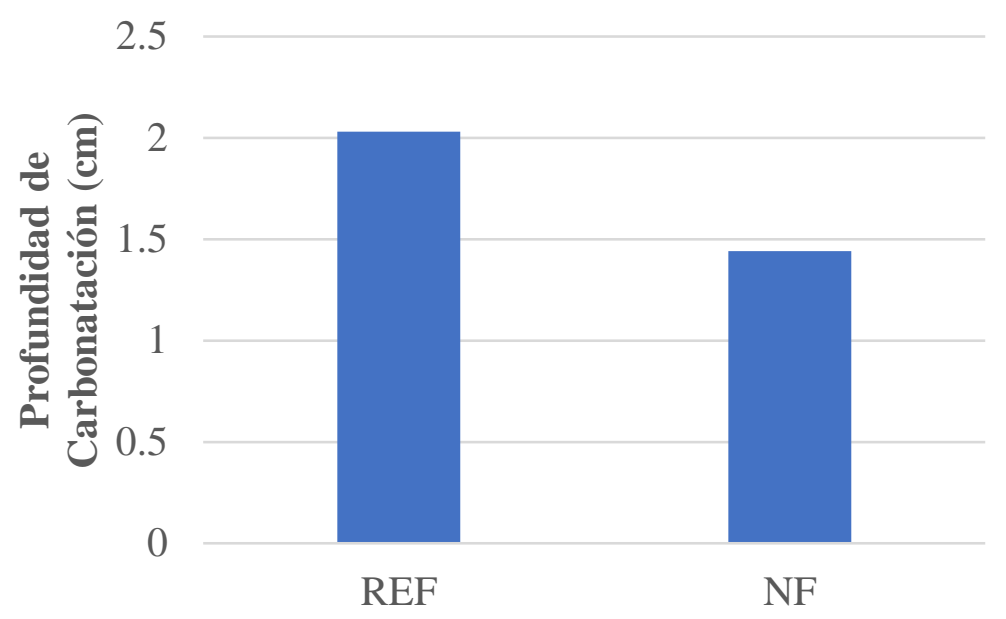

Figura 12. Nivel de carbonatación de núcleos extraídos en rampa.

Con la prueba fenolftaleína se puede medir la profundidad de carbonatación que se tiene en los núcleos extraídos (Figura 12). Analizando los datos, se puede observar que en la muestra tratada hubo un menor avance de la carbonatación. De manera cuantitativa, se reportó una mejoría mayor al 25\% en relación a la resistencia a la carbonatación. Esto cumple la hipótesis planteada en un inicio, al proteger la superficie con una capa hidrofóbica, reduce la humedad que contiene el material. En este sentido, se previene que tome lugar la carbonatación del hidróxido de calcio presente. Es necesario recordar que la rampa contaba ya con 9 años de haber sido construida, aproximadamente. Por lo que era esperado tener un cierto nivel de carbonatación en ambas muestras.

En la Figura 13 se muestran los valores de ángulo de contacto que tiene la parte tratada y la no tratada, luego de los 11 meses de exposición a condiciones naturales. El área tratada ya no presentaba una característica hidrofóbica. Sin embargo, aún mantenía un ángulo de contacto mayor que el de referencia, por lo cual la adherencia del agua era menor que su superficie. Esto se ve reflejado en el seguimiento fotográfico (Figura 14), donde se aprecia que la superficie tratada se mantenía mucho más limpia que la no tratada. La pérdida del efecto hidrofóbico pudo suceder debido al desgaste por el paso de peatones, los rayos UV, los ciclos de mojado y secado por lluvias, la carbonatación, entre otros factores.

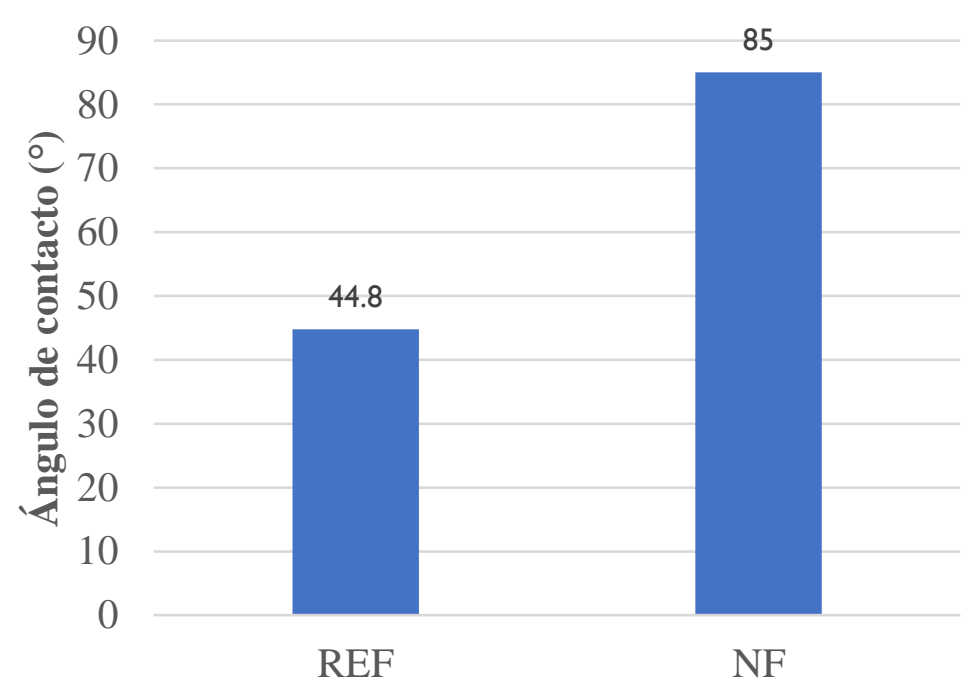

Figura 13. Ángulo de contacto del agua de los núcleos para experimento in situ. 


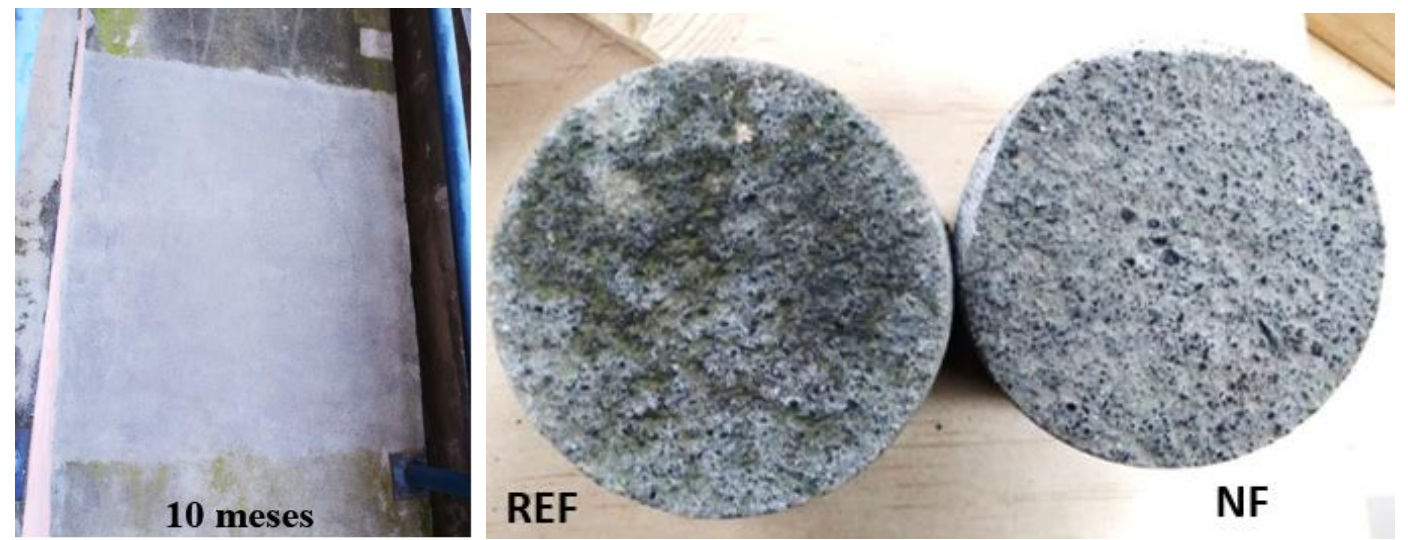

Figura 14. Fotografías luego de 10 meses de exposición, de la rampa usada para el experimento in situ (izquierda) y de los núcleos extraídos de la misma a los 11 meses de exposición (derecha).

Realizando la prueba de determinación de biomasa por peso seco (Tabla 3), se obtuvo que en la muestra de referencia se retiró 4 veces más masa que en el NF. Esto sirve como indicador de cuánto más material orgánico estaba adherido a la superficie de la muestra. El efecto hidrofóbico fungió como inhibidor del crecimiento de microorganismos, evitando que el agua, producto de lluvias o riego, se adhiriera y se presentaran las condiciones necesarias para el crecimiento de estos. Además, la pendiente que tenía la rampa pudo aportar a que el agua fluyera más fácil y se mantuviera limpia la zona tratada.

Tabla 3. Biomasa determinada por seco en experimento in situ.

\begin{tabular}{|c|c|}
\hline Muestra & Peso seco de la biopelícula \\
\hline $\mathrm{REF}$ & $4.3 \mathrm{mg}$ \\
\hline $\mathrm{T} 2$ & $0.9 \mathrm{mg}$ \\
\hline
\end{tabular}

\section{CONCLUSIONES}

1. Las NF presentaron un mejor desempeño al aplicarse en especímenes con $5 \mathrm{~mm}$ de carbonatación, que con los de $0 \mathrm{~mm}$. Pese a que el efecto hidrofóbico se mantenía, la carbonatación se adjudica a las condiciones tan agresivas bajo las cuales fueron expuestos los materiales.

2. Las NF mostraron una reducción en el crecimiento superficial de la Chlorella sp. Sin embargo, se produjo una notable adherencia al sustrato por parte de las microalgas. Esto se les adjudica a las condiciones tan críticas de humedad que se tenían en el biorreactor, produciendo un deterioro en cada uno de los tratamientos.

3. Bajo condiciones reales, la NF mostró la capacidad de inhibir el crecimiento de microorganismos, como moho y líquenes, en el concreto al repeler la humedad de la superficie. Lo que evita que se generen las condiciones necesarias para ser inoculada y colonizada.

4. Bajo las condiciones ambientales de la región, la NF podrían contribuir a la reducción en el avance de la carbonatación de las estructuras de concreto reforzado, por efecto de la reducción de la humedad relativa al efecto hidrofóbico desarrollado en la superficie.

\section{AGRADECIMIENTOS}

Los autores expresan su agradecimiento CONACYT por los recursos otorgados al proyecto CB-285453, así como al Laboratorio de Investigación e Innovación en Materiales de Construcción, al Departamento de Ecomateriales y Energía, al Departamento de Tecnología del Concreto y al Laboratorio de Ingeniería Ambiental por todo el apoyo brindado para el desarrollo de este trabajo. 


\section{REFERENCIAS}

Atta, M., Idris, A., Bukhari, A., Wahidin, S. (2013) Bioresource Technology Intensity of blue LED light: A potential stimulus for biomass and lipid content in fresh water microalgae Chlorella vulgaris. Bioresource Technology., 148: 373-378.

Bastos et al. (2016) Nano-Inclusions Applied in Cement-Matrix Composites: A Review. MDPI: Materials. 9, pp. 5-7.

Bhushan, B. et al. (2010) "Lotus Effect: Surfaces with Roughness-Induced superhydrophobicity, selfCleaning and Low Adhesion" Ohio state University, Ohio.

Cruz Moreno, D. M. A. (2014) "Mejoramiento del efecto barrera en materiales endurecidos de cemento portland mediante una aplicación innovadora de nano-partículas de silicio”. Universidad Autónoma de Nuevo León. pp. 33-80

Cruz-Moreno, D., Fajardo, G., Flores-Vivián, I., Cruz-López, A., Valdez, P. (2017) Tratamiento superficial con nanopartículas base silicio inducido durante el curado: Efecto en la durabilidad de materiales base cemento portland. Revista ALCONPAT, 7 (3), pp. 274-285, DOI: http://dx.doi.org/10.21041/ra.v7i3.239

De Ferri, L. et al. (2011) Study of silica nanoparticles - polysiloxane hydrophobic treatments for stonebased monument protection. Elsevier Masson SAS. 12: 356-363

Fajardo et al. (2014) Innovative application of silicon nanoparticles (SN): Improvement of the barrier effect in hardened Portland cement-based materials. Elsevier: Construction and bluilding materials.

Fernández-Cañete, A. (2013) Estudio de la Hidrofobicidad y Autolimpieza en Materiales con Nanotratamientos Superficiales. Proyecto final de carrera. Universitat Autónoma de Barcelona.

Grengg, C. et al. (2018) Advances in concrete materials for sewer systems affected by microbial induced concrete corrosion: A review. Elsevier: Water Research.

$\mathrm{Li} \mathrm{H}$. et al. (2017) Synthesis and properties of SiO2/P(MMA-BA) core-shell structural latex with siloxanes. Elsevier: Progress in Organic Coatings. 97: 65-73

Márquez, J. F. (2015) “Mecanismos De Biodeterioro Del Concreto Reforzado" Universidad Francisco de Paula Santander. II SEMANA INTERNACIONAL, X SEMANA DE CIENCIA, TECNOLOGÍA E INNOVACIÓN: Cúcuta (Colombia) pp. 118-127

Ortiz-Moreno, M. L., Castillo, C. E. C., Sánchez-Villarraga, J. (2010) Evaluación del crecimiento de la microalga chlorella sorokiniana en diferentes medios de cultivo en condiciones autotróficas y mixotróficas. ORINOQUIA Universidad de los Llanos, Colombia 16: 11-20.

Possan, E. (2010), "Modelagem da carbonatação e previsão de vida útil de estruturas de concreto em meio urbano", Tese de Doutorado em Engenharia, Programa de Pós-Graduação em Engenharia Civil, Universidade Federal do Rio Grande do Sul, Porto Alegre.

Sanjay, S., Latthe et al. (2009) Superhydrophobic silica films by sol-gel co-precursor method. Elsevier: Applied Surface Science. 256: 217-222

Tienda, F. (2019) "Tratamiento superficial con nanopartículas para el control de la carbonatación y el crecimiento de microorganismos en concretos envejecidos" Universidad Autónoma de Nuevo Leon. Zhang, L., Ma, N., Wang, Y., Han, B., Cui, X., Yu, X., Ou, J. (2016) Study on the reinforcing mechanisms of nanosilica to cement-based materials with theoretical calculation and experimental evidence. Journal of Composite Materials. pp. 1-12. 